\title{
ESTUDIOS
}

\section{Proust y la modernidad narrativa ${ }^{1}$}

\author{
Carlos García-Bedoya Maguiña \\ Universidad Nacional Mayor de San Marcos \\ carlos.garcia-bedoya@hotmail.com
}

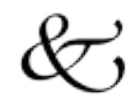

\begin{abstract}
Resumen
Estas reflexiones sobre la obra proustiana están planteadas desde la óptica de la historia literaria. Se estudiará a Proust como un escritor de transición entre las corrientes literarias del siglo XIX y las del siglo XX. Es evidente la amplitud de este asunto, y por ello habrá que restringirse a algunas consideraciones preliminares sobre el tema y concentrar luego los esfuerzos en el examen de la recepción de En busca del tiempo perdido por parte de algunos de sus primeros críticos. El estudio de la recepción permitirá justamente enriquecer esta percepción de Proust como escritor de transición entre dos siglos y sobre todo como una figura clave de la modernidad narrativa.
\end{abstract}

Palabras claves: Proust, Historia literaria, Corrientes literarias, Recepción.

\begin{abstract}
Proustian oeuvre will be approached here from the perspective of literary history. Proust is studied as a transitional author between the literary currents of the nineteenth century and those of the twentieth century. This obviously vast subject will be tackled in a preliminary fashion and then we will proceed with a more thorough examination of the
\end{abstract}

1 Se publica en ocasión de cumplirse cien años de la aparición de Du Côté de chez Swan (Por el camino de Swann), parte inicial de la vasta novela de Proust $\grave{A}$ la Recherche du temps perdu (En busca del tiempo perdido). Según la opinión especializada, la obra de Proust es la novela más larga de toda la literatura mundial. 
reception of Remembrances of Things Past by its early critics. Our focus on reception will allow us to delve into our perception of Proust as a writer between two centuries and as one of the founding fathers of narrative modernity.

Keywords: Proust, Literary history, Literary currents, Reception.

\section{Proust, escritor de transición}

Antoine Compagnon, entre otros, ha tratado el tema ampliamente en su libro Proust entre deux siècles. No se intentará aquí resumir los resultados de las investigaciones sobre asunto tan complejo. Se preferirá, de manera sucinta, presentar primeramente un par de rasgos que vinculan a la obra proustiana con la literatura de fines del siglo XIX, y luego otros dos que la relacionan con las innovaciones vanguardistas de las primeras décadas del siglo XX.

Se considerará, en primer lugar, los entronques sociales de la obra de Proust. Pierre Zima, en su libro Le désir du mythe. Une lecture sociologique de Marcel Proust, recurriendo al método del estructuralismo genético elaborado por Lucien Goldmann, examina los vínculos de la obra proustiana con un fenómeno social muy difundido a fines del siglo XIX: el esnobismo burgués. Es cierto que la aproximación de Zima puede considerarse un tanto reduccionista, pero resulta posible retomar, matizándolas, algunas de sus ideas. Es evidente que la fascinación mundana ante el universo de la aristocracia desempeña un papel importante en En busca del tiempo perdido, y que no se trata de un deslumbramiento meramente personal, sino más bien de una obsesión colectiva de la alta burguesía (y en particular del grupo de los rentistas), deseosa de compartir los remanentes del brillo y del prestigio simbólico de la vieja nobleza, junto a la cual conformaba lo que sociólogos como Veblen han denominado la clase ociosa. Paradójicamente, este fenómeno del esnobismo (en el sentido lato del término) burgués alcanzó amplio predicamento en el momento mismo en que la aristocracia deja de desempeñar un rol social relevante en Francia. El descubrimiento por parte del narrador protagonista de En busca del tiempo perdido de la fragilidad del mito aristocrático es una de las razones (pero sin duda no la única, como parece creer Zima) que refuerza sus convicciones esteticistas. Todo este planteamiento merece sin duda un desarrollo más profundo, por cierto una lectura sociológica de Proust no resultará en modo alguno irrelevante, pero ella no puede realizarse en base a la idea fija de establecer homologías directas entre una obra literaria y la visión del mundo de un grupo social.

El segundo aspecto que se abordará es el del horizonte estético en el cual se sitúa Proust. ${ }^{2}$ Sin ninguna duda, se trata de un horizonte muy decimonóni-

2 El horizonte de producción de su obra, según la terminología de la teoría de la recepción. 
co, y en particular muy fin de siglo. Sus referentes culturales son el simbolismo, el decadentismo, el arte por el arte, la pintura impresionista, la música wagneriana, la pasión ruskiniana por las catedrales góticas, para nada en cambio las corrientes de vanguardia, el cubismo, el expresionismo, el futurismo o el cine (al que parece ignorar completamente). ${ }^{3}$ Compagnon apunta de pasada que "Des surréalistes, à qui la mort d' Anatole France devait bientôt inspirer le pamphlet Un cadavre, Proust ne semble en revanche rien savoir" (Compagnon 1989: 21). ${ }^{4}$ Sus héroes artísticos son Baudelaire o Wagner, no Apollinaire o Picasso. Se han resaltado en especial los lazos de Proust con el simbolismo. Al comentar una obra juvenil de nuestro autor, Les plaisirs et les jours, Levin subraya que "Painting and music were harmonized with poetry, in accordance with the synesthetic ideal of the symbolists" (Levin 1963: 387). ${ }^{5}$ Esa misma ambición de integrar en su obra los aportes de otras prácticas artísticas resulta muy visible en En busca del tiempo perdido. El discurso verbal aspira a transmitir los efectos estéticos de la pintura de Elstir, de la música de Vinteuil o de la actuación de la Berma. Es la misma ambición de producir una obra de arte total que presidía la creación de Wagner. No es casual que los músicos que sirvieron de "modelo" a Vinteuil sean justamente los herederos franceses del arte wagneriano, sobre todo Gabriel Fauré, pero también Claude Debussy o César Franck. El esteticismo casi maniático de En busca del tiempo perdido, ese esfuerzo sistemático por transmutar al nivel de lo sublime los incidentes en apariencia más cotidianos, obedece a las urgencias del horizonte cultural finisecular.

Se examinarán ahora los aspectos de la obra proustiana más orientados hacia el porvenir. En primer lugar, conviene poner de relieve sus paradójicas conexiones con la aspiración totalizadora que se acaba de comentar. En busca del tiempo perdido es una novela en la que con frecuencia la dimensión narrativa no es la dominante. Sin arriesgar demasiado, se podría incluso afirmar que en la mayor parte del texto la historia (en el sentido técnico que tiene el término en la narratología) pasa a un segundo plano, sumergida por un flujo discursivo de naturaleza muy distinta. Ya lo recalcó Harry Levin "There is more descrip-

3 Sin embargo, un fascinante episodio narrado por Painter grafica la posición de Proust como uno de los indudables fundadores de la modernidad artística del siglo XX: se trata de la fastuosa cena ofrecida el 18 de mayo de 1922 (pocos meses antes de la muerte de Proust) por sus amigos los Schiff, grandes mecenas del arte contemporáneo, en la que coincidieron cuatro de las figuras más representativas del arte del siglo XX: Picasso, Stravinski, Joyce y el propio Proust (Painter 1966: II, 422-424).

4 "De los surrealistas, a quienes la muerte de Anatole France debía inspirar pronto el panfleto Un cadáver, Proust no parece en cambio saber nada”. La traducción es mía, en adelante todas las traducciones de otras lenguas lo serán igualmente.

5 "Pintura y música se armonizaban con la poesía, de acuerdo con el ideal sinestésico de los simbolistas". 
tion than narration, and much less illustration than commentary. We seem to be confronting a sequence of essays and memoirs, rather than the usual sort of story" (Levin 1963: 397). ${ }^{6}$ Pareciera que esta subalternidad del componente narrativo es justamente una consecuencia del deseo pan-estético, una resultante de esa voluntad de transmutar lo cotidiano en obra de arte a la que ya se ha aludido. Para alcanzar esa meta, Proust transgrede las fronteras tradicionales de los géneros. Largos pasajes de su obra mayor pueden perfectamente ser leídos como ensayos de crítica artística, otros pasajes, en general más breves, parecen ser poemas en prosa. De tal manera, la aspiración totalizadora de procedencia simbolista o finisecular se encuentra en el origen de una opción escritural muy innovadora, mediante la cual se dinamitan las demarcaciones genéricas al uso. Cabe recordar que esa vocación agenérica o plurigenérica resulta según Bajtin muy característica de la novela moderna. Nos encontramos pues, como resulta evidente, ante un rumbo seguido, con modulaciones muy distintas, por varios de los novelistas más renovadores del siglo XX, como por ejemplo el propio Joyce, cuyo Finnegans wake puede ser leído simultáneamente como una novela y como un gigantesco laboratorio de experimentación verbal, afín por tanto a la poesía.

El segundo lado innovador de En busca del tiempo perdido que se abordará, ha sido el más analizado por la crítica: el tratamiento del tiempo. Problema de suma complejidad también, pero sobre el cual se formularán algunos comentarios, partiendo de las reflexiones de Auerbach en su capital libro Mimesis, reflexiones referidas no solamente a Proust, sino a toda una vertiente de la narrativa de orientación vanguardista. El crítico alemán constata que en la novela contemporánea los sucesos externos de la acción narrativa se ordenan en función de sucesos internos producidos en la propia consciencia de los personajes. El acontecimiento exterior (con frecuencia insignificante en sí mismo) desencadena un proceso interior de reflexión, de recuerdo: es el caso clásico del episodio de la magdalena en Por el camino de Swann, pero también el de los adoquines del patio de los Guermantes que, en El tiempo recuperado (volumen final de la novela proustiana), recuerdan al narrador protagonista los adoquines de esa Venecia que visitó años atrás en compañía de su madre, y por cierto muchos otros episodios de semejante índole. A veces también los acontecimientos externos permiten interpretar ciertos procesos internos aún confusos: así, cuando el narrador protagonista, ya algo envejecido, descubre, gracias a Gilberte, que los caminos que conducen a Tansonville (el lado de Swann) y a Guermantes, caminos que creía tan opuestos, en realidad se terminaban

6 "Hay más descripción que narración, y mucha menos ilustración que comentario. Pareciera que estamos confrontados a una serie de ensayos y memorias, antes que al tipo usual de narración". 
uniendo, logra comprender lo infundado de uno de los mitos que alimentó su infancia y su juventud. Auerbach denomina a este fenómeno de coexistencia de distintas temporalidades en la novela estratificación del tiempo: varias capas temporales se superponen, cada una con su ritmo particular. El tiempo puro del acontecer pierde su importancia, mientras crece la de un tiempo de la subjetividad (contemplativa, reflexiva o rememorativa), o, más precisamente, se configura un nuevo acercamiento a la temporalidad que correlaciona el tiempo del transcurrir (objetivo) con el tiempo vivencial de la conciencia (subjetivo). Esto ayuda a comprender de qué manera una novela como En busca del tiempo perdido debe necesariamente recurrir a formas genéricas como el ensayo o la poesía, que justamente permiten captar mejor ese tiempo interiorizado.

\section{Algunos aspectos de la recepción de En busca del tiempo perdido}

Un enfoque que puede ayudar a comprender la compleja situación de Proust como escritor de transición es el estudio de la recepción de la obra proustiana, en especial el examen de la actitud de la crítica contemporánea ante la publicación de los primeros volúmenes de En busca del tiempo perdido: (1913: Por el camino de Swann; 1919: A la sombra de las muchachas en flor). La recepción crítica de la primera hora presenta la ventaja de poner en evidencia aquello que resultaba desconcertante, para sus primeros lectores, en la obra proustiana, así como aquello que les resultaba familiar.

Para abordar tal problemática, convendrá apoyarse en algunas reflexiones del teórico alemán Hans Robert Jauss (Jauss 1978 y 1988). Jauss reivindica la importancia de la recepción: para él la obra literaria existe sólo en la relación dialógica con el lector. Con demasiada frecuencia se restringió el enfoque de análisis al mero estudio de la producción literaria, en tanto para Jauss "L' histoire de la littérature, c' est un processus de réception et de production esthétiques, qui s' opère dans l' actualisation des textes littéraires par le lecteur qui lit, le critique qui réfléchit et l' écrivain lui-même incité à produire à son tour" (Jauss 1978: 48). ${ }^{7}$

Lo que interesa a Jauss no es sin embargo la recepción subjetiva, impresionista, de un lector particular, su reacción muy personal a una obra. Pretende más bien examinar lo que denomina el horizonte de expectativas del público inicial de una obra. El concepto de horizonte de expectativas designa a un sistema de referencias que guían a una lectura. Es en primer lugar, la experiencia de un género, lo que el lector espera encontrar en una novela, un drama, etc.,

7 "La historia de la literatura es un proceso de recepción y de producción estéticas, que opera mediante la actualización de los textos literarios por el lector que lee, el crítico que reflexiona y el escritor mismo, incitado a su vez a producir". 
experiencia modelada por el conocimiento de la forma y la temática de obras anteriores de similar naturaleza. Por otro lado, es también la percepción de la oposición entre un lenguaje poético (o literario) y un lenguaje práctico: el léxico o las formas sintácticas que se consideran propias de una obra de alta literatura. Finalmente, es la oposición entre mundo imaginario y realidad cotidiana, es decir lo que el lector acepta como posible o verosímil en un determinado género literario. Las obras más innovadores son aquellas que se alejan del horizonte de expectativas de los lectores de una época determinada y que favorecen más bien una modificación de tal horizonte.

Hodson distingue tres etapas en la recepción crítica de la obra de Proust (Hodson 1989). La primera, de 1896 a 1922, abarca la crítica publicada en vida de Proust. La segunda se extiende de la muerte del autor hasta 1931: es la etapa de la consolidación del prestigio europeo de Proust. La tercera va de 1931 a los tiempos en que él escribe su estudio, etapa durante la cual Proust es visto como una figura mayor de la literatura occidental. Hodson recalca que Proust "was never totally ignored and his progress was never really obstructed by disheartening sloughs and trials of strength. Generally speaking, he always elicited some positive response in some area or other" (Hodson 1989: 2). ${ }^{8}$ En efecto, Lhomeau y Coelho recopilan en anexo a su libro Marcel Proust à la recherche d' un éditeur 21 artículos, anuncios, reseñas y entrevistas (además de cuatro publicidades redactadas por el propio Proust) sobre Por el camino de Swann, publicados entre noviembre de 1913 y abril de 1914 en los principales periódicos y revistas de la época. La cantidad es importante para un autor entonces todavía poco conocido, y confirma lo bien fundamentada que resulta la opinión de Hodson.

Los artículos que se van a examinar corresponden pues a la primera etapa de la recepción crítica de Proust. Al comentar los artículos de algunos críticos contemporáneos del autor, se apreciará un desencuentro entre la obra de Proust y las expectativas no sólo del gran público, sino también las de algunos de los críticos más prestigiosos de la época, y cómo incluso aquellos que la acogían con simpatía se esforzaban por encuadrarla en sus parámetros estéticos predilectos. Habrá que limitarse a una pequeña cantidad de artículos que sin embargo resultan bastante representativos de las reacciones críticas a las dos primeras partes de En busca del tiempo perdido.

Se abordará en primer lugar un texto que no fue publicado en la época, y que corresponde a la etapa anterior a la publicación de Por el camino de Swann, es decir al momento en que Proust se encuentra aún en busca de un editor.

8 "nunca fue totalmente ignorado y su progreso nunca se vio realmente obstruido por descorazonadoras depresiones o pruebas de fuerza. Hablando en términos generales, siempre generó reacciones positivas en algún u otro sector". 
Se trata del informe de lectura para la editorial Fasquelle, presentado en 1912 por Jacques Madeleine. ${ }^{9}$ La editorial Fasquelle rechazó la novela, al igual que lo hicieron las editoriales Gallimard y Ollendorf. Resulta importante señalar que en 1912 el primer volumen de la obra proustiana, titulado entonces El tiempo perdido, comprendía también el material que constituye hoy $A$ la sombra de las muchachas en flor.

El juicio de Madeleine es fuertemente negativo. No logra penetrar en el sentido de la obra, a pesar de que disponía de una carta explicativa del autor que acompañaba al manuscrito. Al parecer desesperado, el crítico se lamenta: "Au bout de sept cent douze pages ... on n' a aucune notion de ce dont il s' agit. Qu' est-ce que tout cela vient faire? Qu' est-ce que tout cela signifie? Où tout cela veut-il mener? - Impossible d' en rien savoir!". ${ }^{10}$ Sus críticas pueden sintetizarse en tres reproches principales. En primer lugar, reprocha a Proust la falta de organización de los materiales narrativos. Admite que sólo se trata del primer volumen de una serie que debía entonces abarcar dos o tres. Sin embargo, no advierte en modo alguno el hilo narrativo que une las diversas secuencias del conjunto, percibe simplemente una masa caótica de episodios que no parecen llevar a ningún lado. El segundo reproche apunta a las constantes digresiones, ya sea sobre personajes aparentemente secundarios, ya sea sobre impresiones o sensaciones diversas. Estos reproches permiten apreciar que el texto entonces titulado El tiempo perdido no correspondía a la idea que se hacía Madeleine de lo que debía ser una novela "bien hecha”: unidad de acción, prioridad de la narración sobre la descripción o la reflexión. Madeleine considera que esas múltiples digresiones resultan en ocasiones interesantes, que hay belleza en los detalles, pero que no hay estructura de conjunto. El tercer reproche se refiere a la excesiva longitud, tanto del texto como de la frase de Proust, que no corresponden al ideal de concisión y de claridad que era, al parecer, el ideal de Madeleine. Tales reparos serán frecuentemente retomados por la crítica hostil a la novela. Una observación interesante al final del informe: en opinión de Madeleine, el desarrollo del relato debería lógicamente llevar al joven protagonista a asumir una opción homosexual.

Se examinará enseguida un artículo de Paul Souday. ${ }^{11}$ Souday se sorprende primeramente de la extensión desmesurada de una novela que trata un asunto tan insignificante: "M. Marcel Proust embrasse-t-il dans son grand ouvrage

9 Ese informe se publicó por primera vez en Le Figaro littéraire del 8 de diciembre de1966. Reproducido en Tadié 1971: 10-17, y en Lhomeau y Coelho 1988: 255-262.

10 "Después de setecientas doce páginas ... no se tiene noción alguna de lo que se trata. ¿A qué viene todo esto? ¿Qué significa todo esto? ¿A dónde nos lleva todo esto? - ilmposible saberlo!”. Lhomeau et Coelho 1988: 255.

11 Publicado en Le Temps del 10 de diciembre de 1913. Reproducido en Lhomeau y Coelho 1988: 281-287. 
l' histoire de l' humanité ou du moins celle d' un siècle? Non point. Il nous conte ses souvenirs d' enfance. Son enfance a donc été remplie par une foule d' événements extraordinaires? En aucune façon: il ne lui est rien arrivé de particulier" (Lhomeau y Coelho 1988: 281). ${ }^{12}$ Habrá que excusar la venenosa ironía de Souday, pero nótese que cae de lleno en la trampa autobiográfica y supone que el autor nos está en efecto contando su vida pasada: helo aquí descaminado en busca de una novela confesional, cuando en verdad prima en el texto una construcción reflexiva. Souday insiste luego en la dificultad de la lectura: reprocha a Proust una escritura recargada, atiborrada de cláusulas subordinadas, e incluso de incorrecciones gramaticales, que no eran en verdad sino erratas de imprenta. Pero la acusación más importante es por cierto la de falta de construcción, la sobreabundancia de hechos mínimos: "Il nous semble que le gros volume de M. Marcel Proust n' est pas composé, et qu' il est aussi démesuré que chaotique, mais qu' il renferme des éléments précieux dont l' auteur aurait pu former un petit livre exquis" (Lhomeau y Coelho 1988: 283). ${ }^{13}$ Son, sino las mismas palabras, al menos casi los mismos conceptos que ya se encontraron en el texto de Madeleine. Percibe el lado impresionista de Proust, la importancia de las sensaciones y también la necesidad estética de sublimar la realidad material, pero sólo ve en ello logros parciales, casi siempre perjudicados por su carácter digresivo ("Un amor de Swann” le parece el colmo de esta malhadada tendencia a la dispersión). En conclusión, Souday dice esperar con simpatía la continuación de la obra, pero esperando "y découvrir un peu plus d' ordre, de brièveté et un style plus châtié" (Lhomeau y Coelho: 287). ${ }^{14}$

Se pasará ahora a examinar una tercera (y última) crítica desfavorable, la de Henri Ghéon, ${ }^{15}$ la más dolorosa para Proust, por proceder de una colectividad de la que esperaba y deseaba una acogida favorable (la Nouvelle Revue Française). El reparo mayor se encuentra enunciado ya en la primera línea: la obra de Proust es simplemente una obra de ocio. Es justamente el tipo de prejuicio que llevó a Gide a rechazar la publicación de Por el camino de Swann sin haber leído la obra, como lo confesó por cierto más tarde al propio Proust. Los intelectuales de la Nouvelle Revue Française veían en Proust a un simple mundano, un

12 "¿Abarca el señor Marcel Proust en su gran obra la historia de la humanidad o al menos la de un siglo? Nada de eso. Nos cuenta sus recuerdos de infancia. ¿Su infancia estuvo entonces llena de una multitud de acontecimientos extraordinarios? De ninguna manera: no le ha ocurrido nada especial".

13 "Nos parece que el grueso volumen del señor Marcel Proust no tiene composición, que es tan desmesurado como caótico, pero que encierra elementos valiosos con los que el autor habría podido formar un pequeño libro exquisito".

14 "descubrir en ella un poco más de orden, de brevedad y un estilo más trabajado".

15 Publicada en la Nouvelle Revue Française del 1 de enero de 1914. Reproducida en Lhomeau y Coelho 1988: 307-311. 
asiduo de los salones de la alta sociedad, reprochándole sus vinculaciones con el fenómeno social del esnobismo, al que se ha hecho referencia en la primera parte de este trabajo. Ghéon le echa pues en cara a Proust su pertenencia a esa clase ociosa, de lo que deriva la conclusión (implícita) de que la obra de un autor así no puede ser sino frívola y superficial. Por lo demás sus otras imputaciones son, con pocas variantes, las ya formuladas en las notas de Madeleine y Souday. Aún más que Souday, está convencido de encontrarse ante una novela autobiográfica (o casi memorias) y halla la explicación de la preferencia proustiana por el detalle minucioso en una memoria que no ahorra al lector fragmento alguno de sus recuerdos: "la moindre image de rencontre, le moindre souffle printanier, comme le moindre passant de la rue, ont pris dans sa mémoire une place aussi grande et non moins privilégiée que les plus rares aventures" (Lhomeau y Coelho 1988: 307). ${ }^{16}$ iMemoria tan prodigiosa resultaría sin duda digna de Funes, el protagonista del conocido relato de Borges! Dicha profusión de detalles lleva a Ghéon a constatar el carácter fragmentario de la obra proustiana: "Il écrit des "morceaux». Il place son orgueuil dans le «morceau»: que dis-je? dans la phrase" (Lhomeau y Coelho 1988: 308) ${ }^{17}$ Conclusión necesaria, tal obra carece de unidad, de organización, de composición. Generosamente, Ghéon atribuye todo ello a la sinceridad de Proust, que no le permitiría ahorrarnos el menor detalle de sus recuerdos. Le reprocha también a Proust el no seguir la línea de desarrollo de la caracterización de un personaje, sino más bien presentar sus aspectos contradictorios. Se siente a la vez desconcertado por las libertades que Proust se toma con la cronología, su imprevisible manera de pasar de un tiempo a otro, sin razón aparente. En síntesis, cuestiona a Proust justamente por dos de los rasgos que lo tornan tan interesante para los lectores de comienzos del siglo XXI. En conclusión, anota que "son livre n' est pas un roman, ni un récit, ni même une confession. C' est une "somme», la somme de faits et d' observations, de sensations et de sentiments, la plus complexe que notre âge nous ait livrée" (Lhomeau y Coelho 1988: 310). ${ }^{18}$ Y, si bien le reconoce bellezas parciales, tal conclusión no resulta halagadora para la obra proustiana, considerada amorfa y desprovista de organización.

Lo esencial de las críticas formuladas en los tres artículos que se han comentado confluyen en torno a un aspecto examinado por Compagnon: "Taxer une œuvre d' éparpillement revient en effet à la juger décadente” (Compagnon

16 "la mínima imagen de circunstancia, el mínimo soplo primaveral, así como el más insignificante transeúnte de la calle, han cobrado en su memoria un lugar tan grande y no menos privilegiado que las más peregrinas aventuras".

17 "Escribe «fragmentos». Pone todo su orgullo en el «fragmento»: ¿qué digo? en la frase".

18 "su libro no es una novela, ni un relato, ni siquiera una confesión. Es una "suma», la suma de hechos y de observaciones, de sensaciones y de sentimientos, más compleja que nuestra edad nos haya brindado". 
1989: 39) ${ }^{19}$ Proust tenía plena consciencia de la posibilidad de tal reproche, y sostenía más bien que su obra no era fragmentaria: "c' est au contraire un tout très composé, quoique d' une composition si complexe que je crains que personne ne le perçoive et qu' il apparaisse comme une suite de digressions. C' est tout le contraire" (Compagnon 1989: 43) ${ }^{20}$ Como lo apunta Compagnon, Proust había en verdad llevado al extremo una opción unitaria; para él, "le vrai écrivain est l' homme d' un seul livre, les grands artistes n' ont jamais crée qu' une seule œuvre" (Compagnon 1989: 41). ${ }^{21}$ Desgraciadamente, los peores temores de Proust se concretaron. Es evidente que Madeleine, Souday, Ghéon y tantos otros, habían sido incapaces de captar la estructura unitaria subyacente en el proyecto proustiano, y sólo lograban percibir pura incoherencia. El horizonte de expectativas en el que se situaban esos críticos suponía una acción principal a cuyo servicio debían subordinarse los demás elementos del texto. No lograban captar que el tipo de estructura narrativa a la que estaban acostumbrados carecía de relevancia en una novela que se organizaba más bien de modo musical, alrededor de un conjunto de leitmotive que cobraban plena significación únicamente en función de la conclusión final. Una idea similar permite a Levin explicar la extensión de la frase proustiana: "What rhetoricians would term enumeration, a series of parallels in apposition, branching out to explore divergent areas and yet converging upon a single many-sided idea, this is what extends the length of his sentences" (Levin 1963: 430). ${ }^{22}$ La metáfora de la catedral gótica es la que mejor explica la estructura global de la obra, su composición unitaria o cerrada: como una catedral gótica, En busca del tiempo perdido se organiza en base a una estructura en el fondo simple y simétrica, pero encubierta bajo la proliferación de una multiplicidad de detalles. En la parte final de la obra, El tiempo recuperado, esta comparación entre la estructura del libro y la de la catedral se hace explícita. En una carta a Jean de Gaigneron de 1919, Proust afirma enfáticamente tal paralelismo estructural:

Et quand vous me parlez de cathédrales je ne peux pas ne pas être ému d' une intuition qui vous permet de deviner ce que je n' ai jamais dit à personne et que j' écris ici pour la première fois, c'est que j' avais voulu donner à chaque partie de mon livre le titre: Porche I, Vitraux de l' abside,

19 "Calificar a una obra como desperdigada significaba en efecto juzgarla decadente".

20 "es por el contrario un todo muy compuesto, aunque de una composición tan compleja que temo que nadie lo perciba y que aparezca como una serie de digresiones. Es todo lo contrario". Carta a René Blum de febrero de 1913.

21 "El verdadero escritor es hombre de un sólo libro, los grandes artistas siempre han creado una única obra".

22 "Lo que los retóricos denominarían enumeración, una serie de paralelos en aposición, ramificándose para explorar áreas divergentes, pero convergiendo sin embargo sobre una única idea multifacética: es esto lo que extiende el tamaño de sus oraciones". 
pour répondre d' avance a la critique stupide qu' on me fait de manquer de construction dans des livres où je vous montrerai que le seul mérite est dans la solidarité des moindres parties (Tadié 1986: 241). ${ }^{23}$

Proust transgredía obviamente una serie de normas que los críticos comentados consideraban de absoluta necesidad. Una novela que cuestionaba la primacía de lo narrativo no podía sino desconcertarlos, y eso es justamente lo que hacía Proust al otorgar en su obra un peso similar a los componentes narrativos, descriptivos y reflexivos, violando las tradicionales barreras entre géneros como el ensayo, la novela o la poesía (aspecto al que ya se aludió en la primera parte de este artículo). El propio Proust percibía la condición problemática de su texto, que escapaba a las clasificaciones genéricas. Le escribe a René Blum: "Je ne sais pas si je vous ai dit que ce livre était un roman. Du moins, c' est encore du roman que cela s' écarte le moins" (Proust 1984: 9192). ${ }^{24}$ La experiencia literaria previa de los críticos aludidos exigía también que el personaje tuviera una evolución coherente, y he ahí que Proust se complace en presentarnos visiones contradictorias de sus personajes. De igual manera, la organización del relato debía seguir el hilo cronológico de los acontecimientos, en tanto Proust saltaba a voluntad de un tiempo a otro. Se advierte así en la opción proustiana dos de los rasgos distintivos de la novela contemporánea, destacados por Auerbach en el último capítulo de Mimesis: la presentación de un hecho o de un personaje desde múltiples perspectivas contradictorias (representación pluripersonal de la consciencia), y el predominio del tiempo interior de la consciencia sobre el tiempo exterior de los acontecimientos (estratificación temporal). En todos estos puntos, Proust sobrepasaba el horizonte de expectativas de sus contemporáneos y contribuía a la conformación de la sensibilidad moderna.

Para concluir, se comentarán brevemente las opiniones de otro crítico, esta vez favorable a Proust. Se abordarán dos artículos de Jacques Rivière, ambos posteriores al otorgamiento del premio Goncourt de 1919 a Proust por A la sombra de las muchachas en flor. En el primer artículo, Rivière emprende la defensa de Proust contra los detractores de la decisión de la Academia Goncourt que lo había galardonado. Responde a los que cuestionan la edad de Proust que la juventud de un escritor se mide por la potencialidad innovadora de su obra y subraya que Proust es el gran renovador moderno de la novela sicoló-

23 "Y cuando me habla de catedrales no puedo dejar de emocionarme de una intuición que le permite adivinar lo que nunca he dicho a nadie y que escribo aquí por primera vez, es que quise darle a cada parte de mi libro el título: Pórtico I, Vitrales del ábside, para responder por adelantado a la crítica estúpida que se me hace de carecer de construcción en libros cuyo único mérito, como se lo mostraré, radica en la solidaridad de las menores partes".

24 "No sé si le he dicho que este libro es una novela. Al menos, a pesar de todo, es de la novela de lo que menos se aleja". 
gica. En el segundo artículo, mucho más largo y ambicioso, Rivière construye una imagen de Proust que concuerda con la política literaria de la Nouvelle Revue Française que él dirigía: "Rivière, avec son intelligence et sa finesse, sa culture et ses scrupules, choisit ainsi dans la Recherche ce qui s' accorde avec le projet de la N.R.F. depuis 1913: en réaction contre le symbolisme et l' esprit fin de siècle, fonder un nouveau classicisme" (Tadié 1971: 23). ${ }^{25}$ Comienza sosteniendo que vanguardia política y vanguardia literaria no coinciden, y que Proust se halla a la cabeza de esta última. Luego, procede a oponer a Proust al simbolismo, lo que no carece de un lado paradójico, pues ya se destacaron en la primera parte de este trabajo los múltiples lazos de nuestro autor con el simbolismo. En opinión de Rivière, debido a la influencia simbolista, "Le roman psychologique s' imprègne de lyrisme; il n' est plus une branche de l' étude des passions; il ne sert plus à dessiner des caractères; à de très rares exceptions près, il n' est plus conçu que comme un recueil d' "impressions»" sur l' âme, de "paysages introspectifs»" (Tadié 1971: 26). ${ }^{26}$ Consciente de que tal reparo podría también achacársele a Proust, se apresura a destacar lo que lo separaría de esos simbolistas-impresionistas: Proust es un investigador, un reflexivo, no un visionario. Retomando la dicotomía nietzcheana, sería pues un escritor apolíneo, no uno dionisíaco, un "clásico» (en el sentido intemporal del término) y no un «romántico». Recalca que la introspección proustiana es un mero medio para llegar al conocimiento de los demás, y compara en esto a Proust con Racine. Gracias a Proust, "Notre littérature, un moment suffoquée par l' ineffable, redevient ouvertement ce qu' elle a toujours été: un «discours sur les passions»" (Tadié 1971: 29). ${ }^{27}$ El objetivo de Rivière es evidente: anexarse a Proust en su lucha contra la literatura del siglo XIX, pero no en nombre de una modernidad de vanguardia, sino más bien bajo la bandera de un clasicismo eterno, expresión por excelencia del «alma francesa». No se incidirá en una más amplia discusión de esta interpretación sin duda tendenciosa de Proust. Bastará apuntar que, a pesar de su fervor y su admiración, disminuye el alcance de la obra proustiana para poder encuadrarla dentro de los marcos de referencia de su época, marcos a cuyo estallido En busca del tiempo perdido debía más bien contribuir de modo decisivo.

25 "Rivière, con su inteligencia y su finura, su cultura y sus escrúpulos, escogió así en En busca del tiempo perdido lo que concordaba con el proyecto de la N.R.F. desde 1913: en reacción contra el simbolismo y el espíritu finisecular, fundar un nuevo clasicismo".

26 "La novela sicológica se impregna de lirismo; ya no es más una rama del estudio de las pasiones; ya no sirve para dibujar caracteres; con muy pocas excepciones, sólo se concibe como recopilación de «impresiones»" sobre el alma, de "paisajes introspectivos»".

27 "Nuestra literatura, un momento sofocada por lo inefable, vuelve abiertamente a ser lo que siempre fue: un «discurso sobre las pasiones»". 


\section{Referencias bibliográficas}

AUERBACH, Erich (1982) [1942]. Mimesis. México: Fondo de Cultura Económica.

BAKHTINE, Mikhaï (1987). Esthétique et théorie du roman. París: Gallimard.

COMPAGNON, Antoine (1989). Proust entre deux siècles. París: Éditions du Seuil.

HODSON, Leighton (ed.). (1989). Marcel Proust. The Critical Heritage. Londres / Nueva York: Routledge.

JAUSS, Hans Robert (1978). Pour une Esthétique de la réception. París: Gallimard.

JAUSS, Hans Robert (1988). Pour une Herméneutique littéraire. París: Gallimard.

LEVIN, Harry (1963). The Gates of Horn. A Study of Five French Realists. Nueva York: Oxford University Press.

LHOMEAU, Franck y Alain COELHO (1988). Marcel Proust à la recherche d' un éditeur. París: Olivier Orban.

PAINTER, George D. (1966) [1959]. Marcel Proust. París: Mercure de France, 2 tomos.

PROUST, Marcel (1969) [1913-1927]. À la recherche du temps perdu. Edición de Pierre Clarac y André Ferré. París: Gallimard, 3 tomos.

PROUST, Marcel (1984). Correspondence, Tomo XII, (Philip Kolb, ed.). París: Plon

TADIÉ, Jean-Yves (1971). Lectures de Proust. París: Armand Colin.

TADIÉ, Jean-Yves (1983). Proust. París: Pierre Belfond.

TADIÉ, Jean-Yves (1986) [1971]. Proust et le roman. París: Gallimard.

ZIMA, Pierre (1973). Le désir du mythe. Une lecture sociologique de Marcel Proust. París: Éditions A.-G. Nizet. 